\title{
La simulation numérique des phénomènes de tri granulométrique
}

\author{
J. L. Rahuel actuellement à l'IIHR, Université d'Iowa, USA
}

Ph. Belleudy LHF, Grenoble

\section{Aspects physiques du tri granulométrique et du pavage}

La mobilité des particules est fortement liée à leur diamètre. Lorsque la granulométrie est étendue, les particules sont plus ou moins mobiles. Dans les tronçons soumis à érosion, les petits grains du lit seront emportés plus facilement que les gros et se déposeront les premiers dès que la force tractrice de l'écoulement sera plus faible. Ce tri granulométrique est associé dans les tronçons à érosion au phénomène d'armure qui freine l'érosion du lit : en se dégradant, la rivière découvre des particules suffisamment lourdes pour résister aux écoulements (HARISSON, 1950), (BRAY et CHURCH, 1980), (PARKER et KLINGEMAN, 1982), et en compilation RAHUEL (1985) et SIMON (1986).

Il en résulte à terme une couche protectrice du lit qui prévient toute dégradation ultérieure. $\mathrm{La}$ protection n'est toutefois effective que pour la gamme d'écoulements qui ont façonné cette couche. Un débit plus élevé peut remettre en cause la tenue de cette couche pavée.

Les observations sur les couches pavées statiques (HARISSON, 1950), (GESSler, 1965), (LITTLE et MAYER, 1972), (KLAASSEN, 1987) ont mis en relief certains aspects physiques : le lit pavé est plat, même si au départ il existait des dunes. Il est constitué d'une seule couche de grosses particules disposées en tuiles quand leur forme est allongée.

Une couche pavée est généralement observée au pied des grands réservoirs qui piègent les sédiments. La pente du lit pavé à l'aval du réservoir dépend de l'étendue granulométrique et de la force tractrice des débits restitués par le réservoir. S'il existe dans le lit des particules assez lourdes pour résister à tous les écoulements de la rivière et que la composition granulométrique du lit est relativement homogène dans l'espace, le lit érodé final sera parallèle au lit initial (GESSLER, 1965), (KENNEDY, 1985). En revanche, si l'étendue granulométrique n'est pas suffisante, la pente du lit va diminuer, le lit pivotant autour d'un point fixe à l'aval. Le lit se stabilisera en développant une couche pavée lorsque sa pente permettra aux grosses particules de résister à l'entraînement.

Les différentes observations et résultats d'essais sur canaux de laboratoire conduisent à des conclusions différentes quant à l'évolution de la rugosité d'un lit au cours du pavage. La rugosité peut diminuer lorsque, à un lit initialement couvert de dunes, se substitue un lit plat pavé. En revanche, la rugosité d'un lit initialement plat peut augmenter avec l'accumulation de grosses particules à la surface du lit.

\section{Modélisation}

Le code de calcul CARICHAR permet la modélisation des écoulements et du transport solide dans les rivières (BElleudy et al., 1987), (RAHUEL, 1988). Nous présentons ici la modélisation adoptée pour reproduire les phénomènes de tri granulométrique et de pavage.

\subsection{Classes granulométriques et coefficient de dissimulation}

La courbe granulométrique des matériaux du lit est divisée en $K$ classes homogènes représentées par leur diamètre moyen $d_{k}$. Le débit solide charrié d'équilibre s'écrit sous la forme :

$$
G^{*}=\sum_{k=1}^{K} \beta_{k} \cdot \eta_{k} \cdot G_{k}^{*}
$$

où $G_{k}^{*}$ est le transport solide d'équilibre des matériaux uniformes de diamètre $d_{k}$. Le logiciel CARICHAR utilise actuellement pour exprimer $G_{k}^{*}$ la formule de Meyer-Peter et Muller, $\beta_{k}$ est le pourcentage granulométrique des matériaux de la classe $k$ dans la couche mélangée (la notion de couche mélangée sera introduite 
dans le paragraphe suivant), $\eta_{k}$ est un coefficient de dissimulation qui permet de rendre compte de l'interaction des grains et de simuler l'effet d'armure mobile.

La formule utilisée par KARIM (1982) dans IALLUVIAL est reprise pour $\eta_{k}$ dans CARICHAR :

$$
\eta_{k}=\left(\frac{d_{k}}{d_{50}}\right)^{\alpha}
$$

où $\alpha$ a une valeur différente suivant que $d_{k}$ est supérieur ou inférieur à $d_{50}$.

\subsection{Couche mélangée}

\subsubsection{Objet de la couche mélangée}

L'artifice de la couche mélangée (en abrégé c.m. par la suite) apparaît actuellement incontournable quand on veut considérer l'étendue granulométrique dans un modèle numérique de transport solide.

La c.m. est la partie supérieure du lit. En érosion, cette couche permet de relier les caractéristiques du transport aux caractéristiques granulométriques de couches superficielles du lit. Pour cela, cette couche doit contenir les matériaux susceptibles de partir pendant un pas de temps donné. En dépôt, cette couche permet un stockage momentané des matériaux déposés avant qu'ils ne soient mémorisés sous forme de strates.

En érosion comme en dépôt, la c.m. est, comme son nom l'indique, supposée homogène. Les échanges de matériaux se font entre la c.m. et les matériaux en mouvement (charriage et suspension), d'une part, la c.m. et la sous-couche du lit, d'autre part. Les échanges entre la sous-couche du lit et l'écoulement transitent obligatoirement par la c.m.

L'aspect temporel est essentiel dans l'interprétation physique que l'on peut donner de la c.m. Si l'échelle de temps est très courte, la c.m. s'identifiera à une pellicule superficielle, de l'épaisseur de quelques grains, contenant les particules susceptibles d'être soulevées par une variation de la force tractrice locale. Si l'échelle de temps est moyenne, de l'ordre de la période qu'il faut à une ondulation du lit (ride ou dune) pour parcourir une longueur d'onde ou si l'échelle de temps est plus grande mais qu'il n'y a pas de variations importantes du lit, la c.m. pourra s'identifier à ces ondulations. Si l'échelle de temps est grande et si pendant un pas de temps le lit évolue de façon sensible, la c.m. devra s'identifier à la couche érodée ou déposée.

En conséquence, en modélisation numérique, l'épaisseur de la c.m. et le pas de temps, s'il n'est pas court, ne devront pas être choisis indépendamment l'un de l'autre.

Par ailleurs, la couche pavée apparaît physiquement, dans un contexte d'érosion, comme un état asymptotique de la c.m. La modélisation choisie doit donc pouvoir reproduire correctement le pavage.

\subsubsection{Epaisseur de la couche mélangée et représentation de la couche pavée}

Nous présentons ici deux approches qui se différencient dans la façon dont le pavage est appréhendé. La première approche (par exemple KARIM et al., 1983) comptabilise le nombre de grosses particules qui vont s'immobiliser à la surface du lit, à mesure que la rivière s'enfonce dans ses alluvions. De ce comptage se déduit le pourcentage de surface du lit recouvert par ces particules, à condition qu'elles ne se recouvrent pas. Dans cette approche, l'épaisseur de la c.m. peut être gardée constante ou prise variable, par exemple égale à l'épaisseur des dunes. L'effet de pavage progressif est simulé par une réduction du transport des particules de la c.m. dans la proportion de la surface occupée par les particules immobiles. Lorsque toute la surface du lit est recouverte par les particules immobiles, le transport solide s'annule, le lit est pavé.

La seconde approche (par exemple BORAH et al., 1982) considère la couche pavée comme un état asymptotique de la c.m. Contrairement â la précédente, elle ne nécessite pas la considération d'une couche supplémentaire de pavage. Cette couche est simplement un état particulier de la c.m. Aussi l'épaisseur de la c.m. ne peut plus être gardée constante, si l'on veut reproduire correctement la physique du phénomène. Lorsque le pavage est complet, cette épaisseur doit être ramenée sensiblement à celle d'une particule immobile. Borah et al. proposent la formule suivante pour l'épaisseur de la c.m. $E_{m}$ :

$$
E_{m}=\frac{1}{1-p} \frac{d_{L}}{\sum_{k=L}^{K} \beta_{k}}
$$

où $p$ est la porosité du lit, $L$ l'indice de la plus petite classe immobile (les classes sont indicées de façon croissante pour des diamètres croissants), $d_{L}$ le diamètre de la plus petite classe immobile, $\beta_{k}$ le pourcentage de particules de la fraction granulométrique $k$ dans la c.m.

Cette épaisseur détermine au temps $t$ la quantité de sédiments que doit affouiller la rivière, afin qu'un pavage complet se produise relativement à la limite d'immobilité $L$. Il n'y a alors plus d'échange entre la sous-couche du lit et la c.m. Seules les particules de diamètre $d<d_{L}$ contenues dans la couche du lit d'épaisseur $E_{m}$ au temps $t$ de la phase d'érosion seront érodées à terme. Cette seconde approche nous parait plus physique que la première ; elle a été adoptée dans le code CARICHAR.

Dans chacune de ces approches il faut cependant définir un critère d'immobilité des particules. KARIM et al. (1983) utilisent le critère de Shields, indépendamment de la formule utilisée pour le transport solide. Cette façon de faire a deux inconvénients. Premièrement, les seuils de la formule de Shields et de la formule de transport peuvent être assez différents. Ceci peut entraîner soit des erreurs de continuité solide, soit un saut important dans la valeur du transport solide. Deuxièmement, le critère de Shields est en toute rigueur uniquement applicable au cas de granulométries uniformes ou peu étendues. Il ne peut être appliqué sans ménagement au cas de granulométries étendues.

Dans CARICHAR, on préfère utiliser directement la formule de transport pour déterminer quelles sont les particules immobiles. Si une formule de transport ne comportant pas de seuil (exemple Engelung-Hansen) devait être utilisée, il conviendrait de définir arbitrairement une limite de débit solide en deçà de laquelle les particules sont considérées immobiles. 


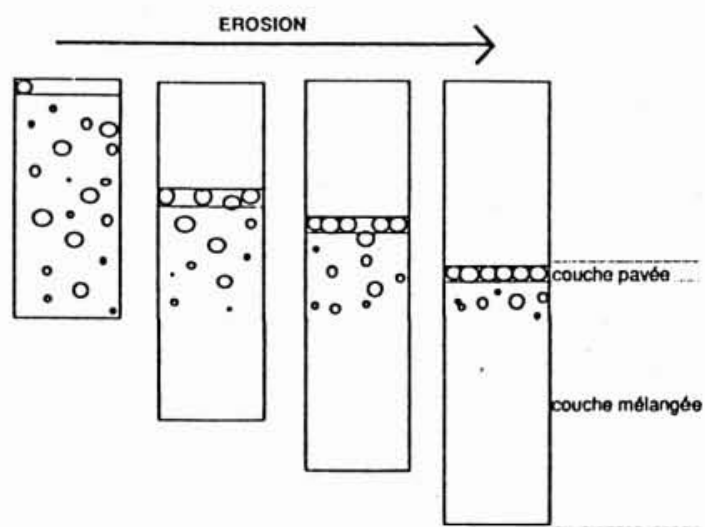

1. PREMIERE APPROCHE : couche pavé indépendante de to couche mélangête

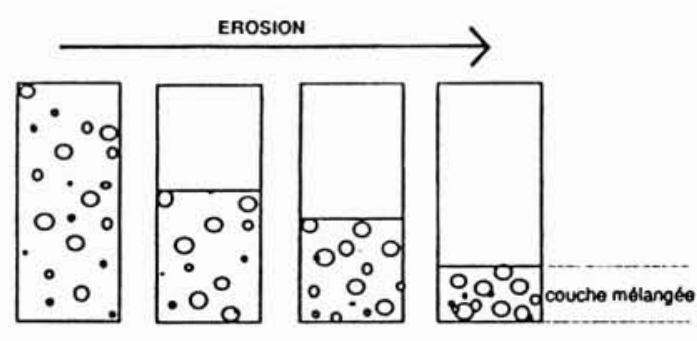

DEUXIEME APPROCHE : la couche pavée est un étal asymptotique de la couche mellangete

2.

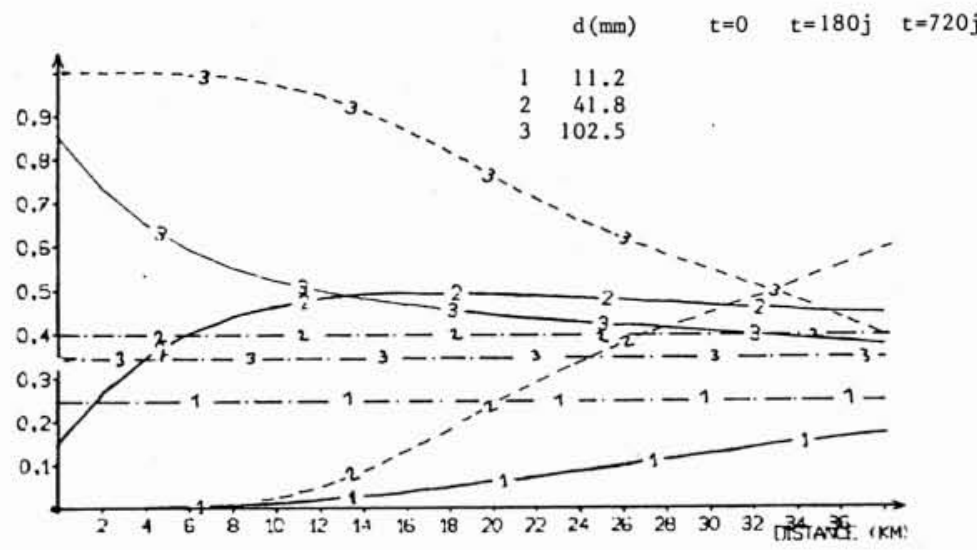

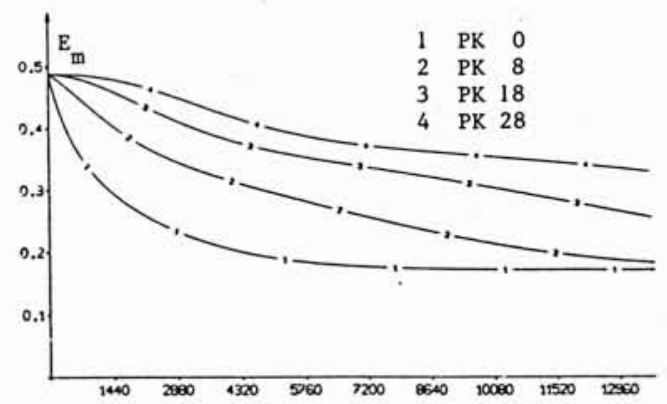

3.

L'application de la formule de Borah peut, dans certains cas, conduire à des épaisseurs de couche mélangée très élevées qui pourraient cacher les variations de taux de transport liés à des strates de composition inhomogènes. Dans le cas de lits qui ne sont pas pavés mais où l'évolution des fonds est lente, la c.m. pourra être identifiée aux dunes ou à une couche de sédiments d'épaisseur équivalente en leur absence. Comme la hauteur maximale des dunes est généralement égale

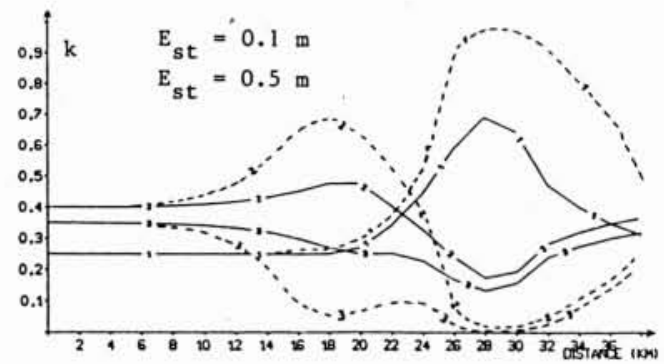

4. 1) $d=11,2 \mathrm{~mm}$; 2) $d=41,8 \mathrm{~mm}$; 3) $d=102,5 \mathrm{~mm}$. 
mesure que le pourcentage de grosses particules augmente dans la c.m., elle finit par prendre le relais de la formule (4).

L'épaisseur de la c.m. égale au diamètre d'une particule immobile est observée physiquement. Dans certains cas, il a toutefois été observé qu'une couche incomplète de ces grosses particules suffisait à stopper toute érosion. (HARRISSON, 1950). Des imbrications particulières de ces particules, par exemple sous forme de couche en tuile, peuvent également se produire. Il est donc raisonnable de compléter la formule (4) par un facteur multiplicatif de réglage.

Dans les sections de la rivière où s'opère un dépôt, la c.m. est identifiée à une couche tampon de stockage. Les variations de la section de la couche mélangée sont égales aux variations de stock alluvial. Ayant défini auparavant une épaisseur de strate $E_{s t}$, on laisse l'épaisseur de la c.m. croître entre $0,5 . E_{s t}$ et $1,5 . E_{s t}$. Lorsque $1,5 . E_{s t}$ est atteint ou dépassé, une nouvelle strate est créée et l'épaisseur de la c.m. est ramenée à $E_{m}-$ $E_{s t}$.

\subsubsection{Bilan de composition de la couche mélangée}

Parmi les équations résolues implicitement pour chaque point de calcul, on trouvera $K$ équations qui expriment chacune la continuité de sédiments d'une classe granulométrique dans le volume de contrôle défini par la c.m. entre deux points de calcul d'indices $j$ et $j+1$.

Cette équation de bilan (5) s'écrit pour la classe $k$ :

$$
\begin{array}{rl}
\beta_{k}^{n+1} & E_{m}^{n+1}=\beta_{k}^{n} E_{m}^{n}+\left(G_{j, k}-G_{j+1, k}\right) \times \\
A \quad B \quad C & B \frac{\Delta t}{\Delta x} \frac{1}{1-p}-\frac{S_{s k} \Delta t}{1-p}+g_{1 k} \frac{\Delta t}{1-p}+ \\
& +\left[\left(z^{n}-E_{m}^{n}\right)-\left(z^{n+1}-E_{m}^{n+1}\right)\right]\left[\varepsilon \beta_{k}^{n}+(1-\varepsilon) \beta_{1 k}\right]
\end{array}
$$

où $(A)$ et $(B)$ sont les volumes de sédiments de classe $k$ dans la c.m. aux temps $n+1$ et $n,(C)$ le volume de sédiments échangé entre le transport charrié et la c.m. pendant $\Delta t,(D)$ le volume de sédiments échangé entre le charriage et la suspension, $(E)$ le volume des apports latéraux, $(F)$ le volume de particules échangé entre la c.m. et la sous-couche du lit (première strate).

$E_{m}$ est l'épaisseur de la c.m., $z$ la cote du fond, $\beta_{k}$ le pourcentage de particules de la fraction granulométrique $k$ dans la c.m., $\beta_{1 k}$ le pourcentage de particules de la fraction granulométrique $k$ dans la première strate du lit, $G_{k}$ le débit solide volumique charrié des matériaux de la classe $k, S_{s k}$ le débit échangé entre les matériaux charriés et les matériaux en suspension, $g_{1 k}$ les apports latéraux de matériaux de classe $k, \varepsilon=0$ ou 1 suivant que la couche mélange absorbe des particules de la première strate ou l'inverse, $\Delta t$ le pas de temps, $\Delta x$ le pas d'espace, $p$ la porosité du lit, $n$ l'indice relatif au temps.

\section{Essais}

Les essais que nous présentons pour illustrer la modélisation par CARICHAR du tri granulométrique et du pavage ont été réalisés sur un bief schématique de rivière présentant sensiblement les caractéristiques du Rhône entre les confluents de l'Isère et de l'Ardèche.
La longueur du bief est de $38 \mathrm{~km}$ et sa pente initiale $S_{0}=0,0007$. Les sections du bief sont supposées rectangulaires ; leur largeur est constante $b=250 \mathrm{~m}$. Le coefficient de rugosité de STRICKLER est supposé constant égal à 36. Le pas d'espace de la discrétisation est dans ces essais de $2000 \mathrm{~m}$.

La courbe granulométrique initiale est décomposée en trois tranches (voir par exemple Belleudy et al., 1987) dont la répartition initiale dans le lit est uniforme telle que :

$$
\begin{array}{rrrrr}
\text { classe } & d_{\min } & \begin{array}{c}
d_{\max } \\
(\mathrm{mm})
\end{array} & d_{\text {moy }} & \begin{array}{c}
\text { fraction } \\
\text { granulométrique } \\
\beta_{k}
\end{array} \\
& & & & \\
1 & 5,0 & 25,0 & 11,2 & 0,25 \\
2 & 25,0 & 70,0 & 41,8 & 0,40 \\
3 & 70,0 & 150,0 & 102,5 & 0,35
\end{array}
$$

\subsection{Formation d'un pavage}

Cet essai représente schématiquement l'évolution d'un bief situé à l'aval d'un barrage qui vient d'être fermé et qui retient tous les sédiments. Le barrage est supposé situé à l'amont immédiat du modèle. Le débit liquide entrant est constant et égal à $4000 \mathrm{~m}^{3} / \mathrm{s}$, le débit solide entrant est nul, le niveau aval est maintenu constant à sa valeur normale. Le pas de temps de la simulation est $\Delta t=48 \mathrm{~h}$.

Seules les deux premières classes granulométriques sont transportées. La formule de l'épaisseur de la c.m. de Borah (éq. (3)) s'impose dès les premiers cycles de calcul. La figure 2 représente la répartition des différentes classes granulométriques dans la c.m. à différents instants. Le pavage du lit est pratiquement réalisé à l'amont du bief au bout de 240 jours et la dégradation du lit est stoppée en ce point où l'enfoncement du lit est alors de $0,317 \mathrm{~m}$ (il est de 1,24 $\mathrm{m}$ dans un essai en granulométrie uniforme à cet instant). La figure 3 montre l'évolution de l'épaisseur de la c.m. en quatre points du modèle. Elle tend vers l'épaisseur de la couche pavée $E_{m}=0,171 \mathrm{~m}$. L'enfoncement du lit tend vers une valeur uniforme, le lit pavé conserve la pente initiale.

\subsection{Strates de dépôt}

On observe cette fois le dépôt des sédiments dans une retenue à l'aval du bief. Le débit liquide est $4000 \mathrm{~m}^{3} / \mathrm{s}$, le débit solide introduit à l'amont est le débit potentiel de chaque classe, le niveau aval est maintenu $10 \mathrm{~m}$ audessus du niveau normal (la profondeur à l'aval est alors de $15,435 \mathrm{~m}$ ).

La variation de composition de la c.m. en dépôt est plus ou moins rapide selon que l'utilisateur a choisi une valeur importante ou faible pour l'épaisseur des strates $E_{s t}$. La figure 4 représente la composition de la c.m. dans le modèle, après 180 jours de simulation, avec respectivement $E_{s t}=0,10 \mathrm{~m}$ et $E_{s t}=0,50 \mathrm{~m}$. Dans le premier cas le stockage de nouvelles strates est fréquent (jusqu'à 19 strates stockées au $p_{k} .28$ en 720 jours contre 4 dans le second cas), la variation de composition est plus rapide. La capacité de transport est alors plus faible à l'aval et les dépôts se produisent donc respectivement 
pour chaque classe granulométrique plus amont avec des strates minces qu'avec des strates épaisses.

\section{Conclusions}

Les résultats obtenus avec le code CARICHAR sont encourageants et justifient a posteriori la plupart des choix effectués et des formulations retenues. La modélisation de la c.m. associée à l'algorithme de gestion des strates conduisent à des résultats crédibles. Ils respectent de plus les bilans volumiques des sédiments. On montre que les résultats obtenus sont peu influencés par les valeurs choisies pour les pas de temps et d'espace, si ce n'est plus précis avec des valeurs plus fines. Les simulations sont très sensibles à la façon dont la courbe granulométrique est décomposée: les résultats sont d'autant plus précis et convergents que le nombre de classes est élevé et un compromis devra être trouvé entre le degré de précision et le coût des calculs.

Les jeux de données disponibles actuellement ne permettent pas la validation de CARICHAR car ils n'ont pas été établis dans l'optique d'un calage d'un code à évolution spatio-temporelle. En particulier on ne connaît souvent que les états initiaux et finaux du système et très peu d'informations existent qui permettent de vérifier le cinétique de mise en place d'un pavage.

\section{Références}

Belleudy Ph., Rahuel J. L., Yang G., CARICHAR mobile bed modelling of graded sediments in unsteady flow, 22nd Congress IAHR, subject $\mathrm{Bb}$ Lausanne, Switzerland, 1987.

Borah D. K., Alfonso C. V., Prasad S. N., Routing graded sediments in streams. Formulations, J. of Hydr. Div., ASCE, Vol. 108, No HY12, 1982.

Bray D. I., ChURCH M., Armored versus paved gravel beds. J. of Hydr. Div., ASCE, Vol. 106, No HY11, 1980.

GESSLER J., The beginning of bed load movement of mixtures investigated as natural armoring in channels. KecK Lab. of hydr. and Water Res., California Inst. of Technology. Pasadena, California, Transl. T5 (revised October 1968), 1965.

HARRISON A. S., The segregation of grain sizes in a degrading bed, M SC thesis, Dept. of Civil Eng., Univ. of California, 1950.

KARIM M. F., KENNEDY J. F., Ialluvial : a computer based flow and sediment routing for alluvial streams and its application to the Missouri river, IIHR rep. No 250. Univ. of Iowa, USA, 1982.

KARIM M. F., Holly F. M., KENNEDY J. F., Bed armouring procedures in Ialluvial and application to the Missouri River, IIHR rep. No 269, Univ. of Iowa, USA, 1983.

KLAASSEN G. J., Armoured river beds during floods, Euromech 215, Genova, Italy, 15-17 September 1987.

LiTtLE W. C., MAYER P. G., The role of sediment gradation on channel armouring, School of Civ. Eng., Georgia Inst. of Technology, Atlanta, 1972.

Parker G., Klingeman P. C., On why gravel bed streams are paved, W Res. Research, Vol. 18, No 5, 1982.

RAHUEL J. L., Modélisation mathématique du pavage en rivière, Rap. de DEA, Univ. Scient. et Médicale de Grenoble, 1985.

Rahuel J. L., Modélisation de l'évolution du lit des rivières alluvionnaires à granulométrie étendue, Thèse Doct. Spécialité Mécanique, Inst. Nat. Polytec. de Grenoble, 1988.

SıMON L., Transport solide de sédiments de granulométrie non uniforme, Mémoire de DEA, Rap. HE/43/86-28, LNH Electr. de France, 1986. 